\title{
On the Number of Linearly Separable Subsets of Finite Sets in $\mathbf{R}^{n}$
}

\author{
T. Gulliksen and A. Hole \\ Department of Mathematics, University of Oslo, \\ Box 1053, Blindern, N-0316 Oslo, Norway \\ \{torg,arneh\}@math.uio.no
}

\begin{abstract}
Let $X$ be a finite set of cardinality $m$ in general position in $\mathbf{R}^{n}$. For $n=3$ we show that if $X$ is in convex position, the number of $k$-sets in $X$ is given by $\Gamma_{k}=$ $2 k(m-k)-m+2$. In general odd dimension we obtain $\sum(-1)^{k} \Gamma_{k}=0$; here convexity is not required.
\end{abstract}

\section{Introduction and Summary}

Let $E$ be an affine space of dimension $n$ over $\mathbf{R}$, equipped with the Euclidean topology. A hyperplane $H \subseteq E$ splits $E$ into a disjoint union of three sets; two open half-spaces and $H$ itself. The open half-spaces are referred to as the half-spaces of $H$. A finite subset $X \subseteq E$ is said to be in general position if no $d$-dimensional affine subspace of $E$ contains $d+2$ elements from $X$. Further, we say that $X$ is in convex position if all points in $X$ are extreme points in the convex hull of $X$. The set $X$ is said to be in convex general position if $X$ is both in convex position and in general position.

Let $X \subseteq E$ be finite, let $H \subseteq E$ be a hyperplane, and let $H^{+}, H^{-}$be the halfspaces of $\bar{H}$. The hyperplane $H$ is said to separate a given subset $A \subseteq X$ if $A \subseteq H^{+}$ and $X \backslash A \subseteq H^{-}$, or alternatively $A \subseteq H^{-}$and $X \backslash A \subseteq H^{+}$. If there is a hyperplane separating $A$, then $A$ is called linearly separable, or just separable. A separable subset $A \subseteq X$ of cardinality $k$ is often called a $k$-set in $X$. The number of $k$-sets in $X$ is written $\Gamma_{k}(X)$. A general introduction to $k$-sets is given in [3].

We are mostly concerned with the case $E=\mathbf{R}^{n}$. By a hyperplane in $\mathbf{R}^{n}$ we always mean an affine hyperplane. The total number of separable subsets in a finite set $X$ of cardinality $m$ in general position in $\mathbf{R}^{n}$ is given by Cover's classical formula $\sum_{k} \Gamma_{k}(X)=$ $2 \sum_{i=0}^{n}\left(\begin{array}{c}m-1 \\ i\end{array}\right)$, see [2]. In this article we obtain more precise information about the integers $\Gamma_{k}(X)$. Our main results are the following two theorems: 
Theorem 1. Let $X$ be a set of cardinality $m$ in convex general position in $\mathbf{R}^{3}$. Then for $1 \leq k \leq m-1$ we have $\Gamma_{k}(X)=2 k(m-k)-m+2$.

Theorem 2. Let $X$ be any finite set in general position in $\mathbf{R}^{n}$. Put $m=|X|$, and assume that $n$ is odd. Then we have $\sum_{k=0}^{m}(-1)^{k} \Gamma_{k}(X)=0$.

It is shown by a counterexample at the end of Section 2 that convexity is not sufficient to ensure invariance of the integers $\Gamma_{k}$ when $n \geq 4$. It also follows from that example that the alternating sum in Theorem 2 is in general not invariant when $n$ and $m$ are both even.

Our method of proof is to deform $X$ in a suitable way into a set $V$ for which we are able to compute the integers $\Gamma_{k}(V)$. We have chosen $V$ to be the set of vertices of a so-called cyclic polytope [3], [4].

The deformation theory required is developed in Section 2, and Corollaries 1 and 2 at the end of that section summarize the invariance results we need. Section 3 contains the calculation of $\Gamma_{k}$ for cyclic polytopes.

In the last section of the paper, we consider a measure of separability which is slightly different from the integers $\Gamma_{k}$. As before, let $X$ be a finite set in $\mathbf{R}^{n}$. By a $k$-hyperplane in $X$ we mean an oriented hyperplane passing through exactly $n+1$ points in $X$, and having exactly $k$ points from $X$ in its positive half-space. For sets $X$ in $\mathbf{R}^{3}, k$-hyperplanes are called $k$-planes. In [1], Clarkson and Shor show that if $X \subseteq \mathbf{R}^{3}$ is in convex general position, then the number of $k$-planes in $X$ is $2(k+1)(m-k-2)$, where $m=|X|$. A proof is also given in [6]. We give an alternative proof based on our "deformation" approach.

\section{Invariance under Deformations}

Let $n, m \geq 1$ be integers, and let $M(n, m)$ be the space of all $n \times m$ matrices over $\mathbf{R}$, with the $\mathbf{R}^{n m}$ topology. We often write matrices from $M(n, m)$ in the form $\left(p_{1}, \ldots, p_{m}\right)$, where $p_{1}, \ldots, p_{m}$ are the columns. Each of these columns is considered as a point in $\mathbf{R}^{n}$. Let $\rho: M(n, m) \rightarrow \wp\left(\mathbf{R}^{n}\right)$ denote the natural map given by $\left(p_{1}, \ldots, p_{m}\right) \mapsto\left\{p_{1}, \ldots, p_{m}\right\}$. (The letter $\wp$ denotes power set.) A matrix $p \in M(n, m)$ is called convex if $\rho(p)$ is a set of cardinality $m$ in convex position in $\mathbf{R}^{n}$. (Note that this implies $p_{i} \neq p_{j}$ for $i \neq j$.) A matrix $p \in M(n, m)$ is called regular if $p_{i} \neq p_{j}$ for $i \neq j$ and the following two conditions are satisfied:

(i) There is no hyperplane $H \subseteq \mathbf{R}^{n}$ containing $n+2$ elements from $\rho(p)$.

(ii) There is at most one hyperplane $H \subseteq \mathbf{R}^{n}$ containing $n+1$ elements from $\rho(p)$.

Finally, a matrix $p \in M(n, m)$ is said to be in general position if $\rho(p)$ is a set of cardinality $m$ in general position in $\mathbf{R}^{n}$. Let

$$
\begin{aligned}
& C(n, m)=\{p \in M(n, m) \mid p \text { is convex }\} \\
& R(n, m)=\{p \in M(n, m) \mid p \text { is regular }\} \\
& G(n, m)=\{p \in M(n, m) \mid p \text { is in general position }\} .
\end{aligned}
$$


Note that $G(n, m) \subseteq R(n, m)$. A continuous map $\sigma:[0,1] \rightarrow M(n, m)$ is called a deformation. If $\sigma(0)=p, \sigma(1)=q$, we say that $\sigma$ is a deformation from $p$ to $q$. We use the notation $\sigma_{j}(t)$ for the $j$ th column of $\sigma(t)$, for $j=1, \ldots, m$. The composition of $\sigma$ with $\rho$ is referred to as the associated map of $\sigma$, and is denoted $\tilde{\sigma}$. Thus $\tilde{\sigma}:[0,1] \rightarrow$ $\wp\left(\mathbf{R}^{n}\right)$. If a deformation $\sigma$ satisfies $\sigma(t) \in R(n, m)$ for all $t \in[0,1]$, and the set $\Omega_{\sigma}:=\{t \in[0,1] \mid \sigma(t) \notin G(n, m)\}$ is finite, then $\sigma$ is called regular. If $\sigma(t) \in C(n, m)$ for all $t$, then $\sigma$ is called convex.

Lemma 1. Let $n \geq 3$. Then we have:

(i) The set $C(n, m)$ is an open, connected subset of $M(n, m)$.

(ii) If $p, q \in G(n, m)$, then there is a regular deformation from $p$ to $q$. If $p, q \in$ $G(n, m) \cap C(n, m)$, then the deformation can be chosen regular and convex.

Proof. (i) Openness is obvious. We show that $C(m, n)$ is path connected. Let $c \in$ $C(n, m)$ be a fixed matrix such that $\rho(c)$ is contained in the unit sphere $S^{n-1}$ in $\mathbf{R}^{n}$. Let $p=\left(p_{1}, \ldots, p_{m}\right) \in C(n, m)$ be arbitrary. We will show that there is a convex deformation $\sigma$ such that $\sigma(0)=p, \sigma(1)=c$.

For each $i \in\{1, \ldots, m\}$, consider a hyperplane $H_{i} \subseteq \mathbf{R}^{n}$ containing $p_{i}$, and having all the other points $p_{j}(j \neq i)$ in precisely one of its half-spaces, say $H_{i}^{-}$. Let $v_{i}$ be the unit normal vector to $H_{i}$ directed into $H_{i}^{+}$, and put $v=\left(v_{1}, \ldots, v_{m}\right)$. Then $v \in C(n, m)$. We have

$$
v_{i} \cdot\left(p_{i}-p_{j}\right)>0 \quad \text { for all } \quad j \neq i .
$$

Observe that we also have

$$
v_{i} \cdot\left(v_{i}-v_{j}\right)>0 \quad \text { for all } j \neq i .
$$

Define $\varphi:[0,1] \rightarrow M(n, m)$ by $\varphi(t)=(1-t) p+t v$. Then $\varphi$ is continuous. To show that $\varphi(t)$ is convex for all $t$, it suffices to show that

$$
v_{i} \cdot\left(\varphi_{i}(t)-\varphi_{j}(t)\right)>0 \quad \text { for all } t \text { and all } j \neq i .
$$

However, this follows readily from (1) and (2), since

$$
v_{i} \cdot\left(\varphi_{i}(t)-\varphi_{j}(t)\right)=(1-t) v_{i} \cdot\left(p_{i}-p_{j}\right)+t v_{i} \cdot\left(v_{i}-v_{j}\right)>0 .
$$

Thus $\varphi$ is a convex deformation with $\varphi(0)=p, \varphi(1)=v$. Since $\rho(v)$ is contained in $S^{n-1}$, it is clear that there is a convex deformation $\psi$ with $\psi(0)=v, \psi(1)=c$. Putting together $\varphi$ and $\psi$, we obtain the desired $\sigma$.

(ii) We prove the convex case, the other case is similar. It follows from standard results in algebraic geometry [5] that the complement of $R(n, m)$ in $M(n, m)$ is closed and contained in a finite union of codimension 2 submanifolds of $M(n, m)$. Hence by (i), $R(n, m) \cap C(n, m)$ is an open, connected subset of $M(n, m)$. Thus there is a deformation $\sigma:[0,1] \rightarrow R(n, m)$ from $p$ to $q$. Now choose a finite covering of the compact set $\sigma([0,1])$ by open cubes in $R(n, m) \cap C(n, m)$ with boundary faces parallell to the standard coordinate hyperplanes in $M(n, m)$. It is clear that we may find a deformation $\sigma^{\prime}:[0,1] \rightarrow R(n, m)$ from $p$ to $q$ whose image is contained in the union of these cubes, 
and which has the additional property that $\sigma^{\prime}(t) \notin G(n, m)$ only for a finite number of $t \in[0,1]$. (Choose $\sigma^{\prime}$ such that only one point in $\tilde{\sigma}^{\prime}(t)$ moves at a time.) Then $\sigma^{\prime}$ is regular and convex.

For the remainder of this section, let $\sigma:[0,1] \rightarrow R(n, m)$ be a fixed regular deformation with associated $\tilde{\sigma}$ and $\Omega_{\sigma}=\left\{t_{1}^{\sigma}, \ldots, t_{s}^{\sigma}\right\} \subseteq(0,1)$. The intervals $\left[0, t_{1}^{\sigma}\right),\left(t_{s}^{\sigma}, 1\right]$, and $\left(t_{i}^{\sigma}, t_{i+1}^{\sigma}\right)$ for all $1 \leq i \leq s-1$ are referred to as the $\Omega_{\sigma}$ intervals.

By a $\sigma$-subset we mean a map $A:[0,1] \rightarrow \wp\left(\mathbf{R}^{n}\right)$ with the property that there is a set $s_{A} \subseteq\{1, \ldots, m\}$ such that $A(t)=\left\{\sigma_{j}(t) \mid j \in s_{A}\right\}$ for all $t \in[0,1]$. If $\left|s_{A}\right|=k$, the map $A$ is called a $\sigma$-subset of cardinality $k$. In that case, we write $|A|=k$. The $\sigma$-subset $B$ such that $s_{B}$ is the complement of $s_{A}$ in $\{1, \ldots, m\}$ is called the complementary $\sigma$ subset of $A$, and is denoted by $A^{\mathrm{c}}$. So $A^{\mathrm{c}}(t)$ is the complement of $A(t)$ in $\tilde{\sigma}(t)$, for all $t$. For given $t$, when we say that $A(t)$ is separable, the meaning is that the set $A(t)$ is separable as a subset of $\tilde{\sigma}(t)$. Further, $A(t)$ is called pseudoseparable if there is some hyperplane $H \subseteq \mathbf{R}^{n}$ such that $A\left(t_{0}\right) \subseteq H \cup H^{+}$and $A^{\mathrm{c}}\left(t_{0}\right) \subseteq H \cup H^{-}$, or alternatively $A\left(t_{0}\right) \subseteq H \cup H^{-}$and $A^{\mathrm{c}}\left(t_{0}\right) \subseteq H \cup H^{+}$. If $H \subseteq \mathbf{R}^{n}$ is a hyperplane, we say that $A(t) \cap H$ is separable in $H$ provided $A(t) \cap H$ is a separable subset of $\tilde{\sigma}(t) \cap H$ in the affine space $H$.

For each $\sigma$-subset $A$, we define the status function $\varphi_{A}:[0,1] \rightarrow\{0,1,2\}$ by letting $\varphi_{A}(t)=0$ if $A(t)$ is separable, $\varphi_{A}(t)=1$ if $A(t)$ is pseudoseparable but not separable, and $\varphi_{A}(t)=2$ if $A(t)$ is not pseudoseparable. We say that $A$ changes status at $t_{0}$ if there is no $\varepsilon>0$ such that $\varphi_{A}$ is constant on $\left(t_{0}-\varepsilon, t_{0}+\varepsilon\right)$. It is easily seen that $\varphi_{A}$ is constant (and equal to 0 or 2 ) on each of the $\Omega$ intervals. If $A$ changes status at $t_{0}$, then since the sets $\left\{t \mid \varphi_{A}(t)=0\right\}$ and $\left\{t \mid \varphi_{A}(t)=2\right\}$ are clearly open in [0,1], we have $\varphi_{A}\left(t_{0}\right)=1$. Moreover, any hyperplane which pseudoseparates $A\left(t_{0}\right)$ must contain at least $n+1$ points from $\tilde{\sigma}\left(t_{0}\right)$, for otherwise we could perturb it to obtain a hyperplane separating $A\left(t_{0}\right)$. However, then, by regularity, the hyperplane pseudoseparating $A\left(t_{0}\right)$ is unique. This hyperplane is called the limit hyperplane of $A$ at $t_{0}$, and is written $H_{\lim }^{A}$.

Lemma 2. Let $n \geq 2$ and $m \geq n+2$. Assume that $A$ changes status at $t_{0} \in \Omega_{\sigma}$, say from $\varphi_{A}(t)=\alpha_{1}$ to $\varphi_{A}(t)=\alpha_{2}$. (That is, $\varphi_{A}(t)=\alpha_{1}$ on the $\Omega_{\sigma}$ interval to the left of $t_{0}$, and $\varphi_{A}(t)=\alpha_{2}$ on the $\Omega_{\sigma}$ interval to the right.) Write $A\left(t_{0}\right)=a_{0} \cup a_{+}$and $A^{\mathrm{c}}\left(t_{0}\right)=a_{0}^{\mathrm{c}} \cup a_{+}^{\mathrm{c}}$ (disjoint unions), where $a_{0}=A\left(t_{0}\right) \cap H_{\lim }^{A}$ and $a_{0}^{\mathrm{c}}=A^{\mathrm{c}}\left(t_{0}\right) \cap H_{\lim }^{A}$. Define two new $\sigma$-subsets $A_{+} \cup A_{0}^{\mathrm{c}}$ and $A_{+}^{\mathrm{c}} \cup A_{0}$ by the conditions $\left(A_{+} \cup A_{0}^{\mathrm{c}}\right)\left(t_{0}\right)=a_{+} \cup a_{0}^{\mathrm{c}}$ and $\left(A_{+}^{\mathrm{c}} \cup A_{0}\right)\left(t_{0}\right)=a_{+}^{\mathrm{c}} \cup a_{0}$. Then we have:

(i) $\tilde{\sigma}\left(t_{0}\right) \cap H_{\mathrm{lim}}^{A}$ is a set of $n+1$ points in general position in the affine space $H_{\mathrm{lim}}^{A}$.

(ii) There are exactly two subsets of $\tilde{\sigma}\left(t_{0}\right) \cap H_{\lim }^{A}$ which are unseparable in $H_{\lim }^{A}$, and these are $a_{0}$ and its complement $a_{0}^{\mathrm{c}}$.

(iii) The four $\sigma$-subsets $A, A^{\mathrm{c}}, A_{+} \cup A_{0}^{\mathrm{c}}$, and $A_{+}^{\mathrm{c}} \cup A_{0}$ are the only $\sigma$-subsets changing status at $t_{0}$, and they change as follows: $A$ and $A^{\mathrm{c}}$ change from $\alpha_{1}$ to $\alpha_{2}$, while $A_{+} \cup A_{0}^{\mathrm{c}}$ and $A_{+}^{\mathrm{c}} \cup A_{0}$ change from $\alpha_{2}$ to $\alpha_{1}$.

Proof. (i) As noted above, the cardinality of $\tilde{\sigma}\left(t_{0}\right) \cap H_{\lim }^{A}$ is $\geq n+1$. By regularity, the cardinality is $\leq n+1$. Now the space $H_{\lim }^{A}$ is $(n-1)$-dimensional. Assume that there is an $(n-2)$-dimensional hyperplane in $H_{\lim }^{A}$ which contains a collection $X$ of $n$ 
points from $\tilde{\sigma}\left(t_{0}\right)$. Choose two points $p_{1}, p_{2}$ in $\tilde{\sigma}\left(t_{0}\right) \backslash X$. (Remember $m \geq n+2$.) Then $X \cup\left\{p_{1}\right\}$ and $X \cup\left\{p_{2}\right\}$ are distinct collections of $n+1$ points from $\tilde{\sigma}\left(t_{0}\right)$ each lying in a hyperplane in $\mathbf{R}^{n}$. Whether these planes coincide or not does not matter, in either case this contradicts the regularity of $\sigma$.

(ii) If $a_{0}$ is separable in $H_{\text {lim }}^{A}$, then we can clearly find a hyperplane $H \subseteq \mathbf{R}^{n}$ which separates $A\left(t_{0}\right)$ in $\tilde{\sigma}\left(t_{0}\right)$. This is impossible, so $a_{0}$ is unseparable in $H_{\text {lim }}^{A}$. Now by (i), $\tilde{\sigma}\left(t_{0}\right) \cap H_{\text {lim }}^{A}$ is a set of $n+1$ elements in general position in the $(n-1)$-dimensional affine space $H_{\mathrm{lim}}^{A}$. Applying Cover's formula, it follows that there are exactly two unseparable subsets. These must then be $a_{0}$ and its complement $a_{0}^{\mathrm{c}}$.

(iii) It follows from (ii) and the definition of $H_{\mathrm{lim}}^{A}$ that all $\sigma$-subsets changing status at $t_{0}$ must be among these four. To show that the four of them change status as stated, observe that $A^{\mathrm{c}}$ obviously must change from $\alpha_{1}$ to $\alpha_{2}$. Since $\tilde{\sigma}(t)$ is in general position for all $t \in\left(t_{0}-\varepsilon\right) \cup\left(t_{0}+\varepsilon\right)$ for some $\varepsilon>0$, it follows from the invariance of the total number of separable subsets (Cover's formula) that there must exist two $\sigma$-subsets changing from $\alpha_{2}$ to $\alpha_{1}$ at the point $t_{0}$. These must be the last two on the list above.

Corollary 1. Let $X, Y$ be finite sets in general position in $\mathbf{R}^{n}$, where $n$ is odd and $|X|=|Y|=m$. Then $\sum_{k=0}^{m}(-1)^{k} \Gamma_{k}(X)=\sum_{k=0}^{m}(-1)^{k} \Gamma_{k}(Y)$.

Proof. The result is trivial if $m \leq n+1$, since all subsets are then separable. It is also easily verified for $n=1$. Now assume $n \geq 3, m \geq n+2$, and choose $p, q \in M(n, m)$ such that $\rho(p)=X, \rho(q)=Y$. By Lemma 1 there is a regular deformation $\sigma$ from $p$ to $q$. Now consider the situation in Lemma 2. Since $n$ is odd, the cardinality $n+1$ of $A\left(t_{0}\right) \cap H_{\mathrm{lim}}^{A}$ is even. Thus $a_{0}$ is even iff $a_{0}^{\mathrm{c}}$ is even. It follows that $\left|A_{+} \cup A_{0}^{\mathrm{c}}\right|$ is even iff $|A|$ is even, and that $\left|A_{+}^{\mathrm{c}} \cup A_{0}\right|$ is even iff $\left|A^{\mathrm{c}}\right|$ is even. Thus the total number of even (resp. odd) separable subsets of $\sigma(t)$ does not change at $t_{0}$. It then follows that these two quantities are invariant for all $t \in[0,1]$, hence so is their difference.

Corollary 2. Let $X, Y$ be two finite sets in convex general position in $\mathbf{R}^{3}$, and assume $|X|=|Y|=m$. Then $\Gamma_{k}(X)=\Gamma_{k}(Y)$ for all $k=0, \ldots, m$.

Proof. Observe again that this is trivial if $m \leq 4$, since all subsets are then separable. Let $m \geq 5$, and choose $p, q \in G(3, m)$ such that $\rho(p)=X, \rho(q)=Y$. By Lemma 1 there is a regular convex deformation $\sigma$ from $p$ to $q$. Let $H_{\lim }^{A}$ be the limit hyperplane of a $\sigma$-subset $A$ at $t_{0}$. The plane $H_{\mathrm{lim}}^{A}$ is two-dimensional, and it contains four points from $\tilde{\sigma}\left(t_{0}\right)$, these lying in general position (Lemma 2). Further, by convexity of $\sigma$ the four points lie in convex position in $H_{\mathrm{lim}}^{A}$ as well. It follows that the two unseparable subsets of $\tilde{\sigma}\left(t_{0}\right) \cap H_{\lim }^{A}$ in $H_{\lim }^{A}$ both have cardinality 2, i.e., $\left|a_{0}\right|=\left|a_{0}^{\mathrm{c}}\right|=2$ in the notation introduced above. So $\left|A_{+} \cup A_{0}^{\mathrm{c}}\right|=|A|$, and $\left|A_{+}^{\mathrm{c}} \cup A_{0}\right|=\left|A^{\mathrm{c}}\right|$. Thus the functions $\Gamma_{k}$ are unchanged at $t_{0}$, and the corollary follows immediately.

By geometrical considerations it can be seen that the invariance of the quantities $\Gamma_{k}$ for sets in convex general position holds in $\mathbf{R}$ and $\mathbf{R}^{2}$ as well. However, we do not have this kind of invariance in $\mathbf{R}^{n}$ for $n \geq 4$, which we now show by a counterexample. Let $n \geq 4$, and consider the points $p_{0}, \ldots, p_{n}, q_{1}, q_{2} \in \mathbf{R}^{n}$ given by $p_{0}=(0, \ldots, 0)$, 
$p_{1}=(1,0, \ldots, 0), \ldots, p_{n-1}=(0, \ldots, 0,1,0), p_{n}=(1, \ldots, 1,2), q_{1}=(1, \ldots, 1)$, and $q_{2}=(1, \ldots, 1,-1)$. Put $X=\left\{p_{0}, \ldots, p_{n}, q_{1}\right\}$ and $Y=\left\{p_{0}, \ldots, p_{n}, q_{2}\right\}$. It is easily verified that both $X$ and $Y$ are in convex general position. Since $|X|=|Y|=n+2$, by Cover's formula there are exactly two unseparable subsets (complementary, of course) in each of the sets. It is easily checked that the unseparable subsets of $X$ are $A=\left\{p_{0}, q_{1}\right\}$ and its complement, while the unseparable subsets of $Y$ are $B=\left\{p_{0}, p_{n}, q_{2}\right\}$ and its complement.

\section{Calculation of $\Gamma_{k}$ for Cyclic Polytopes}

Let $V \subseteq \mathbf{R}^{n}$ be a finite set of points on the "moment curve" $t \mapsto\left(t, t^{2}, \ldots, t^{n}\right)$. The convex hull of $V$ is called a cyclic polytope in $\mathbf{R}^{n}$. The cyclic polytopes are objects with remarkable combinatorial properties [4]. In this section we find recursion formulas facilitating the calculation of $\Gamma_{k}(V)$. At the end of the section we use these results in proving Theorems 1 and 2 stated in Section 1.

Lemma 3. Let $m, n$, and $k$ be positive integers such that $k<m$, and let $V=$ $\left\{\left(t, t^{2}, \ldots, t^{n}\right) \mid t \in\{1,2, \ldots, m\}\right\} \subseteq \mathbf{R}^{n}$. Let $\left(\begin{array}{c}m \\ k\end{array}\right)_{n}$ be the number of separable subsets of $V$ of cardinality $k$, i.e., $\left(\begin{array}{c}m \\ k\end{array}\right)_{n}=\Gamma_{k}(V)$ in the notation above. Then:

(i) $V$ is in convex general position for $n \geq 2$.

(ii) $\left(\begin{array}{c}m \\ k\end{array}\right)_{1}=2$.

(iii) $\left(\begin{array}{c}m \\ k\end{array}\right)_{2}=m$.

(iv) $\left(\begin{array}{c}m \\ k\end{array}\right)_{3}=2 k(m-k)-m+2$.

(v) For $n=2 q$ ( $q$ a positive integer), we have

$$
\left(\begin{array}{l}
m \\
k
\end{array}\right)_{n}=\left(\begin{array}{l}
m \\
k
\end{array}\right)_{n-1}+\left(\begin{array}{c}
k-1 \\
q
\end{array}\right)\left(\begin{array}{c}
m-k-1 \\
q-1
\end{array}\right)+\left(\begin{array}{c}
k-1 \\
q-1
\end{array}\right)\left(\begin{array}{c}
m-k-1 \\
q
\end{array}\right) .
$$

(vi) For $n=2 q+1$ we have

$$
\left(\begin{array}{l}
m \\
k
\end{array}\right)_{n}=\left(\begin{array}{l}
m \\
k
\end{array}\right)_{n-1}+2\left(\begin{array}{c}
k-1 \\
q
\end{array}\right)\left(\begin{array}{c}
m-k-1 \\
q
\end{array}\right) .
$$

Proof. Using Vandermonde's determinant, it is easily seen that the set $V$ is in general position. Put $I=\{1,2, \ldots, m\}$. In order to show that $V$ is in convex position for $n \geq 2$, let $t_{0}, t_{1}, \ldots, t_{r}$ be distinct elements in $I, r \geq 1$, and assume to the contrary that $\left(t_{0}, t_{0}^{2}, \ldots, t_{0}^{n}\right)$ can be written as a convex combination of the vectors $\left(t_{i}, t_{i}^{2}, \ldots, t_{i}^{n}\right)$, where $i=1, \ldots, r$. It would then follow that $\left(t_{0}, t_{0}^{2}\right)$ could be written as a convex combination of $\left(t_{i}, t_{i}^{2}\right)$ for $i=1, \ldots, r$. This is impossible, however, since $\left(t_{i}, t_{i}^{2}\right)$ for $i=0, \ldots, r$ are distinct points on a parabola. So (i) has been established.

The formula in (ii) is trivial, and (iii) follows easily from (ii) and (v). Formula (iv) follows easily from (iii) and (vi). We now concentrate on proving the recursion formula (v).

To see how a hyperplane $c_{0}+c_{1} x_{1}+\cdots+c_{n} x_{n}=0$ splits the set $V$, we first consider its intersection with the curve $\left(t, t^{2}, \ldots, t^{n}\right), t \in \mathbf{R}$. The points of intersection correspond 
to the roots of the polynomial $f(t)=c_{0}+c_{1} t+\cdots+c_{n} t^{n}$, so how the plane cuts the curve corresponds to how the graph of $f$ cuts the $t$-axis. Thus, the way $V$ is split into two separable subsets corresponds to the way the graph of $f$ partitions the set $I$ into a disjoint union of consecutive nonempty subintervals $I=I_{0} \cup \cdots \cup I_{s}$. Observe that since the coefficients of $f$ can be chosen arbitrarily in $\mathbf{R}$, and $f$ may take any degree up to (but not exceeding) $n$, any such partition of $I$ is achievable provided that $s \leq n$.

Note that the two separable subsets of $V$, determined by the hyperplane, correspond to (and have the same cardinality as) the following subsets of $I$ :

$$
A=\bigcup_{\substack{p \text { even } \\ 0 \leq p \leq s}} I_{p} \quad \text { and } \quad B=\bigcup_{\substack{p \text { odd } \\ 0 \leq p \leq s}} I_{p} .
$$

Hence in order to count the separable subsets of $V$ of cardinality $k$, we just have to count the subsets of $I$ of cardinality $k$ having the form of $A$ or $B$ in (3), and sum over all $s$, $1 \leq s \leq n$. Formally, let $\left(\begin{array}{c}m \\ k\end{array}\right)_{A, s}\left(\right.$ resp. $\left.\left(\begin{array}{c}m \\ k\end{array}\right)_{B, s}\right)$ denote the number of subsets of $I$ of cardinality $k$, having the form of $A$ (resp. $B$ ) in (3). Then we have

$$
\left(\begin{array}{l}
m \\
k
\end{array}\right)_{n}=\sum_{s=1}^{n}\left[\left(\begin{array}{c}
m \\
k
\end{array}\right)_{A, s}+\left(\begin{array}{l}
m \\
k
\end{array}\right)_{B, s}\right] .
$$

Observe that

$$
\left(\begin{array}{l}
m \\
k
\end{array}\right)_{B, s}=\left(\begin{array}{c}
m \\
m-k
\end{array}\right)_{A, s}
$$

It follows that

$$
\left(\begin{array}{l}
m \\
k
\end{array}\right)_{n}=\left(\begin{array}{l}
m \\
k
\end{array}\right)_{n-1}+\left(\begin{array}{l}
m \\
k
\end{array}\right)_{A, n}+\left(\begin{array}{c}
m \\
m-k
\end{array}\right)_{A, n}
$$

for $n \geq 2$. We now prove (v). Assume that $s=n=2 q$, where $q \geq 1$. In this case the partition of $A$ in (3) consists of $q+1$ nonempty subintervals, while $B$ consists of $q$ such intervals. Consider the cardinalities $k_{p}=\left|I_{p}\right|, p=0, \ldots, s$. Clearly, $A$ is uniquely determined by the pair of two tuples, on the one hand the $(q+1)$-tuple $\left(k_{0}, k_{2}, \ldots, k_{2 q}\right)$ of positive integers having sum equal to $k$, and on the other hand the $q$-tuple $\left(k_{1}, k_{3}, \ldots, k_{2 q-1}\right)$ of positive integers having sum equal to $m-k$. The first tuple can be chosen in $\left(\begin{array}{c}k-1 \\ q\end{array}\right)$ ways, while the latter can be chosen in $\left(\begin{array}{c}m-k-1 \\ q-1\end{array}\right)$ ways. Hence $A$ can be chosen in $\left(\begin{array}{c}k-1 \\ q\end{array}\right)\left(\begin{array}{c}m-k-1 \\ q-1\end{array}\right)$ ways. So for $n=2 q$ we have

$$
\left(\begin{array}{c}
m \\
k
\end{array}\right)_{A, n}=\left(\begin{array}{c}
k-1 \\
q
\end{array}\right)\left(\begin{array}{c}
m-k-1 \\
q-1
\end{array}\right),
$$

and therefore

$$
\left(\begin{array}{c}
m \\
m-k
\end{array}\right)_{A, n}=\left(\begin{array}{c}
m-k-1 \\
q
\end{array}\right)\left(\begin{array}{l}
k-1 \\
q-1
\end{array}\right) .
$$

Equations (5) and (6) in combination with (4) yields (v). The proof of (vi) is quite similar to the proof of $(\mathrm{v})$ and is left to the reader. 
Remark. The proof above remains valid if the set $I=\{1, \ldots, m\}$ is replaced by an arbitrary set of $m$ distinct elements in $\mathbf{R}$. Thus the lemma gives the number of separable $k$-sets of the set of vertices of an arbitrary cyclic polytope in $\mathbf{R}^{n}$.

We can now prove the two theorems stated in Section 1.

Proof of Theorem 1. The theorem follows immediately from (iv) of Lemma 3 and the invariance property stated in Corollary 2 of Section 2.

Proof of Theorem 2. By Corollary 1 in Section 2, it suffices to prove the theorem in the case $X=V$, where $V$ is as in Lemma 3 above. Let $m, q$, and $k$ be positive integers such that $k<m$. Put $n=2 q+1$. From Lemma 3 we obtain

$$
\left(\begin{array}{l}
m \\
k
\end{array}\right)_{n}=\left(\begin{array}{l}
m \\
k
\end{array}\right)_{n-1}+2\left(\begin{array}{c}
k-1 \\
q
\end{array}\right)\left(\begin{array}{c}
m-k-1 \\
q
\end{array}\right)
$$

and

$$
\left(\begin{array}{l}
m \\
k
\end{array}\right)_{n-1}=\left(\begin{array}{l}
m \\
k
\end{array}\right)_{n-2}+\left(\begin{array}{c}
k-1 \\
q
\end{array}\right)\left(\begin{array}{c}
m-k-1 \\
q-1
\end{array}\right)+\left(\begin{array}{c}
k-1 \\
q-1
\end{array}\right)\left(\begin{array}{c}
m-k-1 \\
q
\end{array}\right) .
$$

Put

$$
a_{k}=\left(\begin{array}{l}
k \\
q
\end{array}\right)\left(\begin{array}{c}
m-k-1 \\
q
\end{array}\right)
$$

for $0<k<n$, and let $a_{k}=0$ for all other values of $k$. Using (7) and (8) it is easy to see that we can write

$$
\left(\begin{array}{l}
m \\
k
\end{array}\right)_{n}=\left(\begin{array}{l}
m \\
k
\end{array}\right)_{n-2}+a_{k-1}+a_{k}
$$

for $0 \leq k \leq m$. It follows that

$$
\sum_{k=0}^{m}(-1)^{k}\left(\begin{array}{l}
m \\
k
\end{array}\right)_{n}=\sum_{k=0}^{m}(-1)^{k}\left(\begin{array}{l}
m \\
k
\end{array}\right)_{n-2}
$$

for $n$ odd, $n \geq 3$. Hence we obtain

$$
\sum_{k=0}^{m}(-1)^{k}\left(\begin{array}{l}
m \\
k
\end{array}\right)_{n}=\sum_{k=0}^{m}(-1)^{k}\left(\begin{array}{l}
m \\
k
\end{array}\right)_{1}
$$

By (ii) of Lemma 3 and the fact that $\left(\begin{array}{c}m \\ 0\end{array}\right)_{1}=\left(\begin{array}{c}m \\ m\end{array}\right)_{1}=1$, we find that the right-hand side of (9) is zero for all $n$, so we have shown that $\sum_{k}(-1)^{k} \Gamma_{k}(X)=0$.

\section{Separability by $k$-Planes}

In [1], Clarkson and Shor determine the number of $k$-planes in a finite set $X$ in convex general position in $\mathbf{R}^{3}$. In this section we give an alternative proof of their result. The proof below uses none of the previous results of this article except for Lemma 1. 
Theorem 3. Let $X$ be a finite set in convex general position in $\mathbf{R}^{3}$. Put $m=|X|$. Then for $0 \leq k \leq m-3$ the number of $k$-planes in $X$ is $2(k+1)(m-k-2)$.

Proof. Let $\sigma$ be a regular convex deformation, and let $k \in\{0, \ldots, m-3\}$ be fixed. Let $\varphi_{k}(t)$ be the number of $k$-planes in the set $\tilde{\sigma}(t)$. We want to show that $\varphi_{k}(0)=$ $\varphi_{k}(1)$.

The situation here is much simpler than the one considered in Section 2. Each change of $\varphi_{k}(t)$ along the way must correspond to some point moving through the plane spanned by three other points. Thus it is clear that the function $\varphi_{k}$ will be piecewise constant on $[0,1]$, and that if $t_{0}$ is a point of discontinuity for $\varphi_{k}$, then $\tilde{\sigma}\left(t_{0}\right)$ is not in general position. By regularity of $\sigma$, there are only a finite number of such points. Let $t_{0}$ be one of them. Again by regularity, there is only one plane $H_{\text {lim }}$ containing more than three points from $\tilde{\sigma}\left(t_{0}\right)$, and moreover $H_{\text {lim }}$ contains exactly four points. By convexity, these four points lie in convex general position in the affine space $H_{\text {lim }}$. Now for each $\sigma$-subset $A$ of cardinality 3 , define $H_{A}:[0,1] \rightarrow \wp\left(\mathbf{R}^{3}\right)$ by letting $H_{A}(t)$ be the nonoriented plane spanned by $A(t)$. The map $H_{A}$ is called a moving plane of $\sigma$. Since $H_{\text {lim }}$ contains four points from $\tilde{\sigma}\left(t_{0}\right)$, there are exactly $\left(\begin{array}{l}4 \\ 3\end{array}\right)=4$ moving planes $H$ of $\sigma$ such that $H\left(t_{0}\right)=H_{\text {lim }}$. Denote these by $H_{1}(t), \ldots, H_{4}(t)$. It is clear that among all the moving planes of $\sigma$, only these four planes can possibly contribute to any change in $\varphi_{k}$ at $t_{0}$. To see what happens to them, let $\varepsilon>0$ be sufficiently small for $\tilde{\sigma}(t)$ to be in general position when $t \in\left[t_{0}-\varepsilon, t_{0}\right) \cup\left(t_{0}, t_{0}+\varepsilon\right]$. Assume that $H_{\lim }$ has $r$ points from $\tilde{\sigma}\left(t_{0}\right)$ on one side, where $0 \leq r \leq m-4$. It is then easy to see that at time $t=t_{0}-\varepsilon$, two of the planes (say $H_{1}$ and $H_{2}$ ) will have $r+1$ points on one side and $m-r-4$ on the other, while $\mathrm{H}_{3}$ and $\mathrm{H}_{4}$ will have $r$ points on one side and $m-r-3$ on the other. At $t=t_{0}+\varepsilon$, however, $H_{1}$ and $H_{2}$ will have $r$ points on one side and $m-r-3$ on the other, while $H_{3}$ and $H_{4}$ will have $r+1$ points on one side and $m-r-4$ on the other. Thus $\tilde{\sigma}\left(t_{0}-\varepsilon\right)$ and $\tilde{\sigma}\left(t_{0}+\varepsilon\right)$ must necessarily have the same number of $k$-planes. It follows that $\varphi_{k}(0)=\varphi_{k}(1)$, and Lemma 1 then gives us invariance of the number of $k$-planes for all finite sets of fixed cardinality in convex general position in $\mathbf{R}^{3}$. We now calculate these invariants by considering our specific example.

Let $\gamma: \mathbf{R} \rightarrow \mathbf{R}^{3}$ be given by $\gamma(t)=\left(t, t^{2}, t^{3}\right)$, and let $V=\gamma(\{1, \ldots, m\})$. Then $V$ is in convex general position in $\mathbf{R}^{3}$. Consider an oriented plane $H$ in $\mathbf{R}^{3}$ with the equation $c_{0}+c_{1} x+c_{2} y+c_{3} z=0$. The condition that $\gamma(t)$ is on the positive side of $H$ (given a suitable choice of sign) translates into the condition that

$$
f(t):=c_{0}+c_{1} t+c_{2} t^{2}+c_{3} t^{3}>0 .
$$

Now $H$ is a $k$-plane in $V$ (for some $k$ ) iff $f$ is zero at exactly three distinct points in $\{1, \ldots, m\}$, and conversely these three zeros determine each $k$-plane in $V$ uniquely up to orientation. So we just have to count how many combinations of three zeros there are such that $f$ is $>0$ on exactly $k$ points in $\{1, \ldots, m\}$. Remembering that $f$ is a general third-degree polynomial, it is clear that all $\left(\begin{array}{c}m \\ 3\end{array}\right)$ conceivable combinations of zeros can be achieved. By symmetry between the functions $f(t)$ and $f(m-t+1)$ (which never determines the same oriented plane, including when $k=(m-3) / 2)$, we may assume 
that the zeros $a, b, c \in\{1, \ldots, m\}$ are such that $a<b<c$ and

$$
\begin{array}{lll}
f(j)>0 & \text { for all } & j<a, \\
f(j)<0 & \text { for all } & a<j<b, \\
f(j)>0 & \text { for all } & b<j<c, \\
f(j)<0 & \text { for all } & j>c .
\end{array}
$$

Let $k$ be fixed. To specify such a combination of zeros $a, b, c$ giving exactly $k$ points where $f$ is positive, the two parameters $a$ and $c$ can be chosen independently subject to the restrictions $1 \leq a \leq k+1$ and $k+3 \leq c \leq m$. So there are $k+1$ choices for $a$, and $m-k-2$ choices for $c$, giving a total of $(k+1)(m-k-2)$ combinations. Multiplying by two (remember the symmetry) gives the result.

It can be shown (by the type of example we considered at the end of Section 2) that the number of $k$-planes in a finite set $X$ of fixed cardinality in convex general position is not invariant for dimensions $n \geq 4$. The details are omitted.

\section{References}

1. K. Clarkson and P. Shor, Applications of random sampling to computational geometry, II, Discrete Comput. Geom. 4 (1989), 387-421.

2. T. M. Cover, Geometrical and statistical properties of systems of linear inequalities with applications in pattern recognition, IEEE Trans. Electron. Comput., 14 (1965), 326-334.

3. H. Edelsbrunner, Algorithms in Combinatorial Geometry, Springer-Verlag, Berlin (1987).

4. B. Grünbaum, Convex Polytopes, Wiley (1967).

5. J. Harris, Algebraic Geometry, Springer-Verlag, New York (1992).

6. M. Sharir, $k$-Sets and random hulls, Combinatorica, 13 (1993), 483-495.

Received January 18, 1995, and in revised form January 21, 1997. 